\title{
Dopamine Receptor Dop1R2 Stabilizes Appetitive Olfactory Memory through the Raf/MAPK Pathway in Drosophila
}

\author{
Huan Sun, ${ }^{1}$ TTomoki Nishioka, ${ }^{2}$ Shun Hiramatsu, ${ }^{1}$ Shu Kondo, ${ }^{3}$ Mutsuki Amano, ${ }^{2}$ Kozo Kaibuchi, ${ }^{2}$ \\ Toshiharu Ichinose, ${ }^{1,4,5,6 *}$ and Hiromu Tanimoto ${ }^{1 *}$ \\ ${ }^{1}$ Graduate School of Life Sciences, Tohoku University, Aoba-Ku, Sendai 980-8577, Japan, 2Department of Cell Pharmacology, Graduate School of Medicine, \\ Nagoya University, Nagoya 466-8550, Japan, ${ }^{3}$ Invertebrate Genetics Laboratory, National Institute of Genetics, Mishima 411-8540, Japan, ${ }^{4}$ Frontier \\ Research Institute for Interdisciplinary Sciences, Tohoku University, Sendai 980-8578, Japan, ${ }^{5}$ Center for Transdisciplinary Research, Niigata University, \\ Niigata 950-2181, Japan, and 'Department of Neuropharmacology, Nagoya City University, Nagoya 467-8603, Japan
}

In Drosophila, dopamine signaling to the mushroom body intrinsic neurons, Kenyon cells (KCs), is critical to stabilize olfactory memory. Little is known about the downstream intracellular molecular signaling underlying memory stabilization. Here we address this question in the context of sugar-rewarded olfactory long-term memory (LTM). We show that associative training increases the phosphorylation of MAPK in KCs, via Dop1R2 signaling. Consistently, the attenuation of Dop1R2, Raf, or MAPK expression in KCs selectively impairs LTM, but not short-term memory. Moreover, we show that the LTM deficit caused by the knockdown of Dop1R2 can be rescued by expressing active Raf in KCs. Thus, the Dop1R2/Raf/MAPK pathway is a pivotal downstream effector of dopamine signaling for stabilizing appetitive olfactory memory.

Key words: dopamine receptor; Drosophila; memory stabilization; Raf/MAPK pathway

\section{Significance Statement}

Dopaminergic input to the Kenyon cells (KCs) is pivotal to stabilize memory in Drosophila. This process is mediated by dopamine receptors like Dop1R2. Nevertheless, little is known for its underlying molecular mechanism. Here we show that the Raf/MAPK pathway is specifically engaged in appetitive long-term memory in KCs. With combined biochemical and behavioral experiments, we reveal that activation of the Raf/MAPK pathway is regulated through Dop1R2, shedding light on how dopamine modulates intracellular signaling for memory stabilization.

\section{Introduction}

Dopamine signaling in the central nervous system is crucial for memory and behavioral adaptation. In addition to well described roles in conveying reinforcement signals and motivation (Busto

\section{Received July 4, 2019; revised Feb. 3, 2020; accepted Feb. 6, 2020}

Author contributions: M.A., K.K., T.I., and H.T. designed research; H.S., T.N., and S.H. performed research; S.K. contributed unpublished reagents/analytic tools; H.S., T.N., S.H., T.I., and H.T. analyzed data; H.S., T.I., and H.T. wrote the paper.

This study was supported by Ministry of Education, Culture, Sports, Science and Technology-Japan/JSPS KAKENHI (17H05545, 17H01378, 15 K14307 to H.T.; 16 K20919 to T.I.; 17 H01380 to K.K.) and Strategic Research Program for Brain Sciences (H.T., K.K., M.A.). The Otsuka Toshimi Scholarship Foundation provided financial support during the study (to H.S.). We thank Nobuhiro Yamagata, Kei Ito, and Ayako Abe for technical and/or instructional supports at inception stage. We also thank Tomoyuki Miyashita, Yukinori Hirano, Bruce A. Edgar, and Minoru Saitoe for technical assistance and/or fly sharing, some of which were not shown in this article. In addition, we thank the Janelia FlyLight Project Team, the Bloomington Drosophila Stock Center, and the Kyoto Stock Center for fly strain provision.

*T.I. and H.T. contributed equally to this work.

The authors declare no competing financial interests.

Correspondence should be addressed to Hiromu Tanimoto at hiromut@m.tohoku.ac.jp or Toshiharu Ichinose at toshiharu.ichinose.c1@tohoku.ac.jp.

https://doi.org/10.1523/JNEUROSCl.1572-19.2020

Copyright $\odot 2020$ the authors et al., 2010; Puig et al., 2014), dopamine signaling has been shown to stabilize nascent memory traces. This role is shown to be conserved across animal phyla ranging from arthropoda to chordata (O’Carroll et al., 2006; Rossato et al., 2009; Plaçais et al., 2012; Musso et al., 2015; Takeuchi et al., 2016).

Studies using olfactory aversive memory of Drosophila melanogaster particularly provide solid evidence regarding how dopaminergic modulation stabilizes memory. Paired presentations of odor and electric shocks increase the activity of specific dopamine neurons (DANs) with oscillating intracellular calcium concentrations during consolidation (Plaçais et al., 2012). Consistently, the blockade of these DANs in the early retention period impedes long-term memory (LTM) consolidation, while activation of them facilitates it (Plaçais et al., 2012, 2017). Intriguingly, the same pair of DANs is also engaged in the consolidation of sugarrewarded LTM with activity oscillation (Musso et al., 2015; Pavlowsky et al., 2018). Another class of DANs is required in the early consolidation phase for appetitive LTM as well (Ichinose et al., 2015). Similarly in courtship suppression learning, LTM consolidation is mediated by specific DANs (Krüttner et al., 2015). No- 
tably, all of these DANs innervate a neural structure called the mushroom body (MB).

$\mathrm{MB}$ intrinsic neurons, Kenyon cells (KCs), express all four of the following dopamine receptors (Croset et al., 2018): Dop1R1 and Dop2R, the functional counterparts of mammalian dopamine $\mathrm{D}_{1}$ and $\mathrm{D}_{2}$ receptors, respectively (Sugamori et al., 1995; Hearn et al., 2002); and two other invertebrate-specific receptors, Dop1R2 and DopEcR (Mustard et al., 2005; Srivastava et al., 2005). Indeed, three of these receptors have been reported to function in KCs for consolidated memories, which are Dop1R1 (Qin et al., 2012; Ichinose et al., 2015; Krüttner et al., 2015), Dop1R2 (Musso et al., 2015; Plaçais et al., 2017), and Dop2R (Scholz-Kornehl and Schwärzel, 2016). Nevertheless, how dopamine regulates intracellular signaling in KCs to stabilize memory remains sparsely understood.

We here examined the role of different dopamine receptors in KCs for appetitive memory. Furthermore, with combined biochemical, genetic, and behavioral characterization, we found that Dop1R2 stabilizes appetitive memory through the Raf and mitogen-activated protein kinase (MAPK) pathway in KCs. As MAPK is a well characterized hub molecule to mediate synaptic plasticity, cytoskeleton reorganization, or transcriptional change, our work provides a critical link between the extracellular dopaminergic modulation and the intracellular signaling in the context of LTM processing.

\section{Materials and Methods}

Fly culture and strains. Flies were cultured on the standard cornmeal medium at $24^{\circ} \mathrm{C}$ in a $12 \mathrm{~h}$ light/dark cycle. Canton-S was used as wildtype fly strain. MB010B-GAL4 (Aso et al., 2014) was obtained from Janelia Farm Research Campus. MBSW-GAL4 is the $P\{M B$-switch $\} 12-1$ line (Mao et al., 2004). Fly strains obtained from the Bloomington Drosophila Stock Center include the following: UAS-IVS-mCD8::GFP (catalog \#32189; Pfeiffer et al., 2010); GMR57C10-GAL4 (catalog \#39171); GMR13F02-GAL4 (catalog \#48571; Jenett et al., 2012); UASDop1R1.RNAi (P\{TRiP.HMC02344\}; catalog \#55239); UAS-Dop1R2. RNAi (P\{TRiP.HMC02893\}; catalog \#51423); UAS-Dop2R.RNAi (P\{TRiP.HMC02988\}; catalog \#50621); UAS-DopEcR.RNAi (P\{TRiP. JF03415\}; catalog \#31981); UAS-Raf.RNAi [1] (P\{TRiP.HMC04133\}; catalog \#55863); UAS-Raf.RNAi [2] (P\{TRiP.HMC03854\}; catalog \#55679) and UAS-MAPK.RNAi (P\{TRiP.HMS00173\}; catalog \#34855). UAS-Raf.GOF (catalog \#106635; Brand and Perrimon, 1994) was acquired from the Kyoto Stock Center. Venus-tagged endogenous dopamine receptor strains were generated as described previously (Kondo et al., 2020).

Behavior assay. Female and male flies, 5-9 d of age after eclosion, were subjected to behavioral assay. Flies were wet starved before and after conditioning so that the mortality reaches $\sim 10 \%$ at the test. Apparatus and protocol for the appetitive conditioning and test were described previously (Ichinose and Tanimoto, 2016). Unless specially stated, appetitive differential conditioning was performed, during which one odor [conditioned stimulus $(\mathrm{CS}+)$ ] was reinforced by dried sucrose (SigmaAldrich) reward, while the other odor (CS-) was not (Figs. 1-5, 6A, B). Exposure of CS + or CS - lasted for $1 \mathrm{~min}$, with a $1 \mathrm{~min}$ interval in between. 4-Methylcyclohexanol (MCH; Sigma-Aldrich) and octan-3-ol (OCT; Merck), diluted in paraffin wax oil to $2 \%$ and $1.2 \%$, respectively, were used as odors. At the test, flies were transferred into a T-maze, where two odors were sucked from the two ends. Flies were allowed to freely explore between two arms and were recorded by cameras (FFMV03M2M and GS3-U3-51S5M, FLIR) for $2 \mathrm{~min}$. The fly number in each arm was automatically counted using an ImageJ (National Institutes of Health) custom-made macro. Based on these numbers, the learning index (Tempel et al., 1983) was calculated for every second. An average of learning indices in the last $60 \mathrm{~s}$, when the performance usually reaches the plateau (Ichinose and Tanimoto, 2016), was used as a single data point. As for single-odor learning (Fig. $6 C-G$ ), the protocol followed exactly that of differential learning, except that $1.2 \%$ OCT was replaced with the pure paraffin wax oil during both the conditioning and test phase. For unpaired stimuli presentation (Fig. 5), the sucrose reward was presented first for $1 \mathrm{~min}$, followed by a rest interval of $2 \mathrm{~min}$. Then $2 \% \mathrm{MCH}$ and $1.2 \%$ OCT were presented, each for $1 \mathrm{~min}$, with another $1 \mathrm{~min}$ interval in between.

Western blot analysis. Starved flies were subjected to appetitive differential conditioning described above and were deep frozen by liquid nitrogen at the indicated time points afterward. The "naive" groups were similarly starved and mock trained without odors and sugar reward, and subsequently were frozen. Around 200 frozen flies were put into a tube and were vortexed to separate heads and bodies. The mixture was poured onto a prechilled metal mesh (710 $\mu \mathrm{m}$ aperture; TS-50-20, Tokyo Screen) to sieve the bodies, and subsequently onto another mesh (425 $\mu \mathrm{m}$ aperture; TS-50-20, Tokyo Screen) to sieve the heads. The frozen heads were ground to powder by using Multibeads shocker (Yasui Kikai) and were resuspended in lysis buffer $(20 \mathrm{~mm}$ Tris/ $\mathrm{HCl}, \mathrm{pH} 7.4 ; 1 \mathrm{~mm}$ EDTA; 1 mm dithiothreitol; $150 \mathrm{~mm} \mathrm{NaCl}$; 1\% igepal CA-630; PhosSTOP (Sigma-Aldrich); proteinase inhibitor cocktail (Sigma-Aldrich). The samples were sonicated and centrifuged at 20,000 $\times g$ for $20 \mathrm{~min}$. Supernatants were collected and used as the protein extracts. Concentrations of the protein extracts were measured by BCA assay (Wako). For Western blot analysis, $15 \mu \mathrm{g}$ of each sample was loaded to $10 \%$ acrylamide gel and subjected to SDS-PAGE. Separated proteins were transferred to PVDF membranes (Immobilon-FL, Millipore). The membranes were blocked with Blocking one-P (Nacalai Tesque) for $1 \mathrm{~h}$ and probed with primary antibodies [rabbit anti-phospho-p44/42 MAPK (catalog \#4370, Cell Signaling Technology), or rabbit anti-p44/42 MAPK (catalog \#4695, Cell Signaling Technology)]. Goat anti-rabbit Alexa Fluor 680 antibody (catalog \#A20984, Thermo Fisher Scientific) was used as a secondary antibody. Bands of antibody binding were detected and quantified using Odyssey CLx imaging system (LI-COR).

Immunohistochemistry. For Venus-tagged dopamine receptors and MAPK immunohistochemistry (Figs. 1, 3), brains were dissected in PBS, followed by the fixation in $4 \%$ paraformaldehyde in PBS for $2 \mathrm{~h}$ at room temperature. Brains were subsequently washed three times and incubated in blocking solution (PBS with 3\% goat serum and $0.1 \%$ Triton $\mathrm{X}-100)$. Then brains were incubated with antibodies in PBS containing $1 \%$ goat serum and $0.1 \%$ Triton $\mathrm{X}-100$. The following primary antibodies were used at the indicated dilution: rabbit anti-GFP (1:1000; catalog \#A11122, Thermo Fisher Scientific), rabbit anti-p44/42 MAPK (1:500; catalog \#4695, Cell Signaling Technology). Alexa Fluor 488 goat antirabbit (1:1000; catalog \#A11034, Thermo Fisher Scientific) was used as the secondary antibody. As for phosphorylated MAPK (pMAPK) immunohistochemistry (Fig. 5), flies were instantly anesthetized on ice and brains were dissected in ice-cold fixative (PBS containing 4\% paraformaldehyde and $15 \%$ saturated picric acid solution). Then brains were fixed in the preceding fixative for $2 \mathrm{~h}$ on ice. Washing and blocking were performed similarly as stated above, but on ice instead. Rabbit antiphospho-p44/42 MAPK (1:500; catalog \#4370, Cell Signaling Technology) and Alexa Fluor 568 goat anti-rabbit (1:1000; catalog \#A11036, Thermo Fisher Scientific) were used as primary and secondary antibodies, respectively, at the indicated dilution. Images were obtained using an Olympus FV1200 confocal microscope, acquired at the same time periods under the identical microscope setting. For counting pMAPK-positive cells, the experimenter was blinded regarding the sample identity: samples were randomized by another experimenter so that they could not be identified by the one who counted the cells. The average number from the two brain hemispheres was used as a single data point (Fig. 5B).

Drug administration. RU486 (mifepristone; Sigma-Aldrich) was administrated with food for $2 \mathrm{~d}$, then with water during food deprivation. RU486 was removed for the last $2 \mathrm{~h}$ before conditioning and after conditioning to avoid any nonspecific effects (Mao et al., 2004). RU486 was dissolved in ethanol $(10 \mathrm{mg} / \mathrm{ml})$ and mixed with melted food or water in a final concentration of $200 \mu \mathrm{M}$ (Mao et al., 2004). The same amount of ethanol was added to the food or water for the control groups.

Data analysis and statistics. Statistical analyses were performed on GraphPad Prism 6 (GraphPad Software). Data were analyzed with parametric statistics: one-sample $t$ test or one-way ANOVA followed by Sidak's multiple-comparisons test, when the assumption of normal distribution 
A
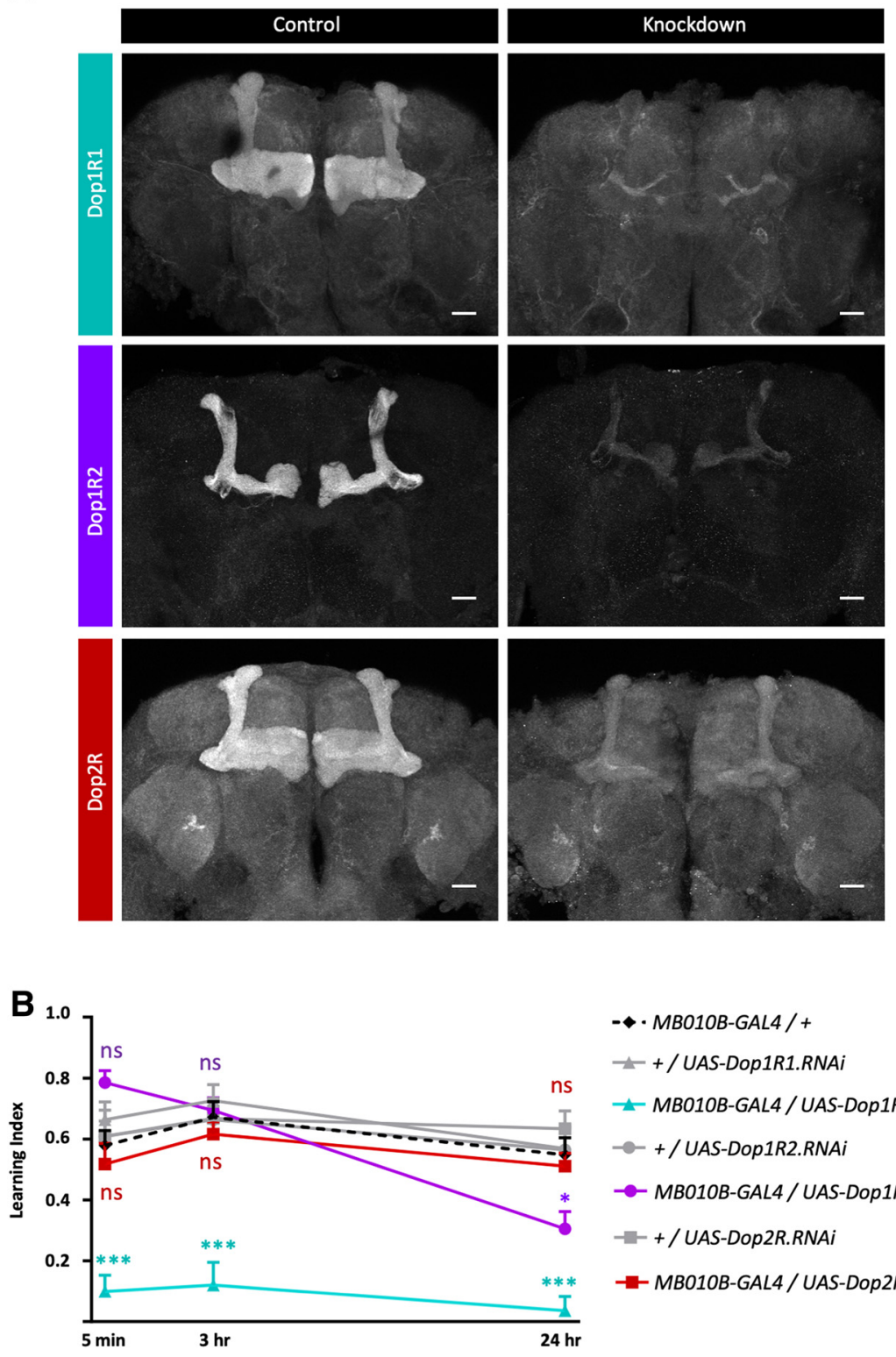

$-\nleftarrow \cdot M B 010 B-G A L 4 /+$

-+ / UAS-Dop1R1.RNAi

- MB010B-GAL4 / UAS-Dop1R1.RNAi

$--+/$ UAS-Dop1R2.RNAi

- MB010B-GAL4 / UAS-Dop1R2.RNA

-+ + /UAS-Dop2R.RNAi

- MBO1OB-GAL4 / UAS-Dop2R.RNAi

Figure 1. Differential engagement of dopamine receptors in short-term and long-term olfactory appetitive memory. $\boldsymbol{A}$, Verification of the transgenic RNAi. Each dopamine receptor is knocked down in Kenyon cells and the endogenous protein is visualized by tagging the Venus yellow fluorescent protein. The Venus protein is stained using antibodies described in Materials and Methods. Genotypes in the control and the knockdown groups are as follows: (top) Dop1R1-Venus,R13F02-GAL4/+ and Dop1R1Venus,R13F02-GAL4/UAS-Dop1R1.RNAi; (middle) Dop1R2-Venus, R13F02-GAL4/+, and Dop1R2-Venus, R13F02-GAL4/UASDop1R2.RNAi; (bottom) Dop2R-Venus/+ „R13F02-GAL4/+ vs Dop2R-Venus/+ „R13F02-GAL4/UAS-Dop2R.RNAi. R13F02-GAL4 labels $\alpha / \beta, \alpha^{\prime} / \beta^{\prime}$ and $\gamma \mathrm{KCs}$. Z-projection images containing the horizontal and vertical lobes are shown. Scale bars, $20 \mu \mathrm{m} . \boldsymbol{B}$ Each of the three dopamine receptors is knocked down in $\mathrm{KC}$, and appetitive memory is measured at different retention times. Attenuation of Dop 1R1 expression severely impairs $5 \mathrm{~min}, 3 \mathrm{~h}$, or $24 \mathrm{~h}$ appetitive memory $\left(5 \mathrm{~min}: F_{(6,51)}=8.17, p<0.0001 ; 3 \mathrm{~h}\right.$ : $\mathrm{H}=24.34, p=0.0005, \mathrm{df}=6 ; 24 \mathrm{~h}: F_{(6,65)}=12.14, p<0.0001 ;$ MB010B-GAL4/UAS-Dop 1R1.RNAi vs GAL4 and UAS controls: 5 min: $p<0.0001$ to both; $3 \mathrm{~h}: p=0.0018$ and $p=0.0002$, respectively; $24 \mathrm{~h}: p<0.0001$ to both). Attenuation of Dop 1R2 expression impairs $24 \mathrm{~h}$ memory but leaves $5 \mathrm{~min}$ and $3 \mathrm{~h}$ memory intact (MB010B-GAL4/UAS-Dop1R2.RNAivs controls: $5 \mathrm{~min}: p=$ 0.073 and $p=0.1726$, respectively; $3 \mathrm{~h}: p>0.9$ to both; $24 \mathrm{~h}: p=0.0174$ and $p=0.0072$, respectively). Memory disturbance in Dop2R expression-attenuated flies is not detected (MB010B-GAL4/UAS-Dop2R.RNAi vs controls: 5 min: $p>0.9$ to both; 3 h: $p>$ 0.9 to both; $24 \mathrm{~h}: p>0.9$ and $p=0.588$, respectively). $n=8-12$. Bars and error bars, mean \pm SEM, respectively. ${ }^{*} p<0.05$, ${ }^{* * *} p<0.001$. ns, Not significant $(p>0.05$ ).

(Shapiro-Wilk normality test) and homogeneity of variance (Bartlett's test) were not violated. Otherwise, nonparametric statistics, Kruskal-Wallis test followed by Dunn's multiple-comparisons test, were performed. The significance level of statistical tests was set to 0.05 .

\section{Results}

Dopamine receptors in the MB are differently engaged in short-term and long-term appetitive memory

To examine the role of the dopamine receptors for appetitive memory retention, we systematically characterized the requirement of them from short-term memory (STM) to LTM. To this end, we knocked down each of them in KCs by transgenic RNAi, using a KC-specific split-GAL4 strain MB010B-GAL4 that labels $\alpha / \beta, \alpha^{\prime} / \beta^{\prime}$ and $\gamma$ KCs (Vogt et al., 2014). The transgenic RNAi effectively reduced the level of Dop1R1, Dop1R2, and Dop2R in the MB, visualized by the endogenous receptors tagged with the Venus yellow fluorescent protein (Fig. 1A; Kondo et al., 2020). Because the RNAi against DopEcR did not show a strong reduction (data not shown), we excluded the receptor from the following behavioral analysis. Then we tested the sugarrewarded memory at $5 \mathrm{~min}$ (STM), $3 \mathrm{~h}$ [often referred to as middle-term memory (MTM)], and $24 \mathrm{~h}$ (LTM; Krashes and Waddell, 2008; Colomb et al., 2009) after conditioning (Fig. 1B). Attenuation of Dop1R1 expression in KCs severely impaired appetitive memory regardless of the retention time tested here. In contrast, attenuation of Dop1R2 expression in KCs left STM and MTM intact but impaired LTM, consistent with previous observations (Musso et al., 2015). We did not observe significant differences when Dop2R expression was attenuated. These results suggest distinct roles of dopamine receptors Dop1R1 and Dop1R2 for mediating dopaminergic appetitive reinforcement and stabilizing memory, respectively.

\section{The Raf/MAPK pathway stabilizes appetitive memory in $\mathrm{KCs}$}

To probe into intracellular signaling under these dopamine receptors, we referred to the results of our previous phosphoproteomic analysis in the mouse striatum (Nagai et al., 2016a,b). This analysis identified many upregulated or downregulated phosphorylation sites in response to the application of dopamine receptor agonists (Nagai et al., 2016a,b). The emergence of MAPK and numerous putative MAPK phosphorylation target proteins, along with Raf (MAPKKK) inspired us to hypothesize that the Raf/MAPK pathway as an effector of dopamine signaling.

To investigate the role of Raf in appetitive memory maintenance, we downregulated Raf expression in KCs and tested memory retention. Attenuation of Raf expression using two independent RNAi insertions caused a selective deficit in $24 \mathrm{~h}$ memory, while leaving 5 
min and $3 \mathrm{~h}$ memories intact (Fig. 2A), suggesting the selective role of Raf in LTM. This retention time course was similar to that of the knockdown of Dop1R2 in KCs (Fig. 1B).

The evolutionary conserved oncogene Raf acts as a core component in multiple cellular processes during development (Leicht et al., 2007). To address the role of Raf in adult but not in developing KCs, we used the RU486-inducible transgenic expression in the MB (MBSW-GAL4) to restrict the Raf knockdown spatially and temporally (Roman et al., 2001; Mao et al., 2004). The adult-specific Raf attenuation impaired 24 h memory (Fig. 2C), in contrast to the intact STM (Fig. 2B) or LTM in the control group without RU486 application (Fig. 2D).

Next, we questioned whether the downstream effector MAPK is also required for LTM. Consistent with the selective LTM impairment of the Raf knockdown, attenuating the expression of the rolled $(r l)$ gene encoding MAPK in adult KCs significantly impaired $24 \mathrm{~h}$ memory, without perturbing 5 min memory (Fig. $3 A-C$ ). Immunohistochemistry of MAPK confirmed the reduction of the protein in the MB (Fig. 3D). Collectively, these results reveal that the Raf/MAPK pathway is required in KCs for appetitive memory stabilization.

Appetitive learning induces Dop1R2-

dependent MAPK activation

The selective requirement of Dop1R2, Raf, and MAPK for appetitive LTM prompted us to hypothesize that the Raf/ MAPK pathway is activated under the control of Dop1R2 upon appetitive learning. Electric shock conditioning was reported to induce MAPK diphosphorylation, thereby activating the kinase (Moressis et al., 2009; Pagani et al., 2009; Miyashita et al., 2018; Zhang et al., 2018). To examine whether appetitive training induces MAPK activation, we monitored the time course of pMAPK by sampling fly heads in different time points after appetitive conditioning (Fig. 4A). Quantification of the pMAPK ratio to the total MAPK (tMAPK) revealed significant increases immediately after learning (i.e., 1, 3 , and $10 \mathrm{~min}$ ), which returned to the basal level after $30 \mathrm{~min}$. Interestingly, this experience-dependent increase of MAPK phosphorylation disappeared by neuronal knockdown of Dop1R2 (Fig. 4B).

To monitor learning-dependent MAPK activation in $\mathrm{KCs}$, we next performed immunohistochemistry of pMAPK. By counting the number of pMAPK-positive
A

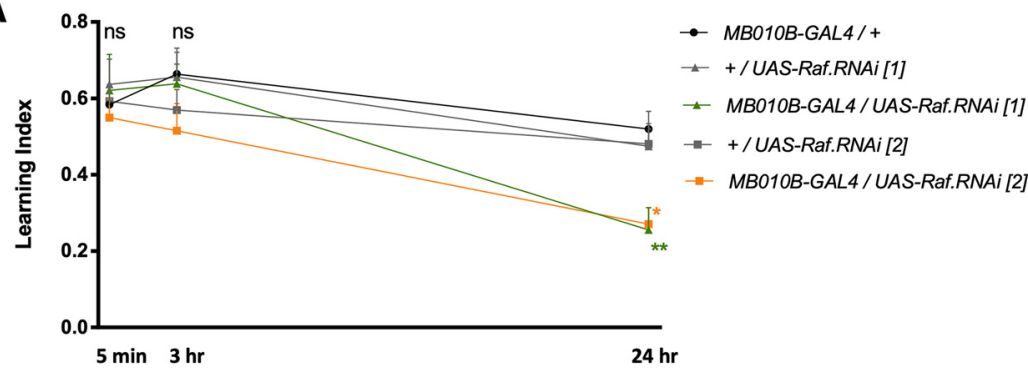

B

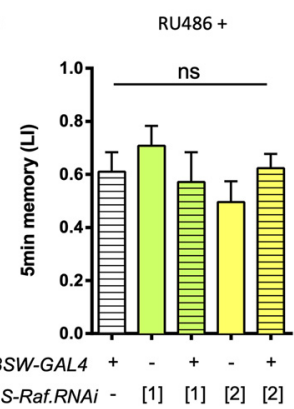

C

RU486 +

D

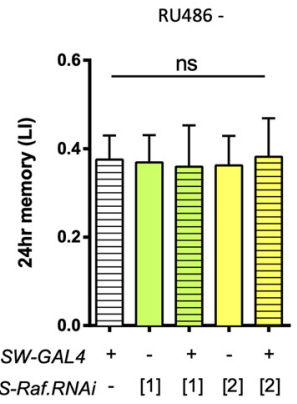

Figure 2. Raf is specifically required for appetitive long-term memory. $A$, Attenuation of Raf expression in KCs impairs $24 \mathrm{~h}$, but not 5 min or $3 \mathrm{~h}$ memory $\left(24 \mathrm{~h}: F_{(4,71)}=6.581, p=0.0001 ;\right.$ MBO10B-GAL4/UAS-Raf.RNAi[1] vs GAL4 and UAS controls: $p=0.0013$ and $p=0.0062$, respectively; MBO10B-GAL4/UAS-Raf.RNAi[2] vs controls: $p=0.0031$ and $p=0.0239$, respectively, $n=11-17$; $\left.5 \mathrm{~min}: F_{(4,44)}=0.3068, p=0.8719 ; 3 \mathrm{~h}: F_{(4,45)}=1.091, p=0.3723, n=9-12\right) . B, C$, Knockdown of Raf in adult KCs using RU486-induced MBSW-GAL4 exhibits intact 5 min memory $\left(B: F_{(4,35)}=0.919, p=0.4640, n=8\right)$, while showing $24 \mathrm{~h}$ memory defect (C: $F_{(4,53)}=6.173, p=0.0004 ;$ MBSW-GAL4/UAS-Raf.RNAi[1] vs controls: $p=0.0191$ and $p=0.0150$, respectively; MBSW-GAL4/UAS-Raf.RNAi[2] vs controls: $p=0.0113$ and $p=0.0033$, respectively, $n=9-14$ ). $\boldsymbol{D}$, Control flies without RU486 feeding attain intact $24 \mathrm{~h}$ memory $\left(F_{(4,47)}=0.01589, p>0.9, n=9-11\right)$. Bars and error bars, mean \pm SEM, respectively. ${ }^{*} p<$ $0.05,{ }^{* *} p<0.01$. ns, Not significant $(p>0.05)$.

A

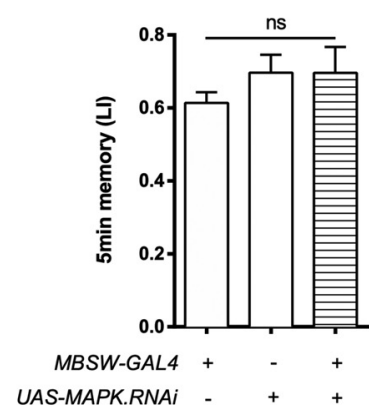

B

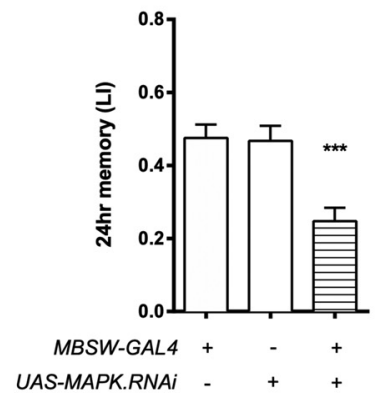

C

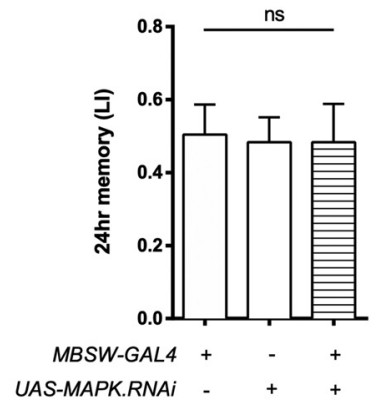

D

MBSW-GAL4 / UAS-MAPK.RNAi
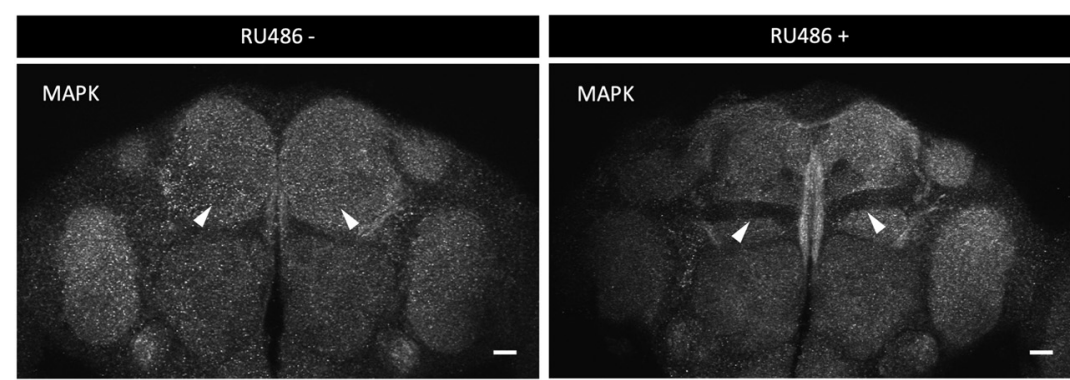

Figure 3. MAPK is specifically required for appetitive long-term memory. $\boldsymbol{A}, \boldsymbol{B}$, Knockdown of MAPK in adult KCs leaves 5 min memory intact $\left(\boldsymbol{A}: F_{(2,21)}=0.8162, p=0.4556, n=8\right)$, while impairs $24 \mathrm{~h}$ memory $\left(\boldsymbol{B}: F_{(2,42)}=11.39, p=0.0001, n=15\right) . \boldsymbol{C}$, Flies without RU486 feeding show intact $24 \mathrm{~h}$ memory $\left(F_{(2,21)}=0.019, p>0.9, n=8\right) . \boldsymbol{D}$, MAPK expression is suppressed in the MB when the RNAi is induced in adult KCs (right, RU486+), compared with the control (left, RU486-). Arrowheads point to the $\beta$ lobes. Scale bars, $20 \mu \mathrm{m}$. Bars and error bars, mean \pm SEM, respectively. ${ }^{* *} p<0.001$. ns, Not significant $(p>0.05)$. 
A
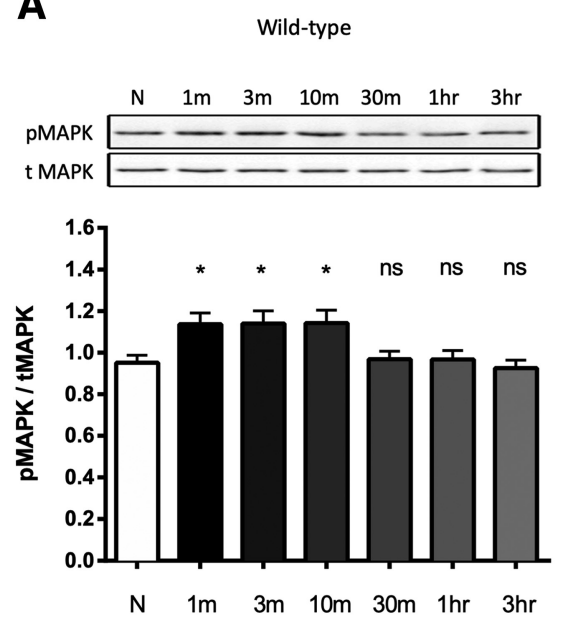

B
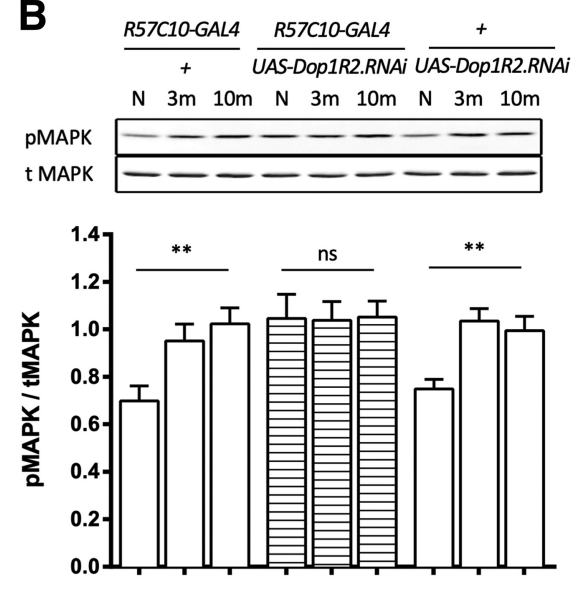

N $3 m 10 m \quad N 3 m 10 m \quad N 3 m 10 m$

Figure 4. Experience-induced MAPK phosphorylation requires Dop1R2.A, Phosphorylation of MAPK before and after appetitive conditioning in wild-type flies. Representative Western blot shows pMAPK and tMAPK in naive (N) and conditioned flies at different time points (from $1 \mathrm{~min}$ to $3 \mathrm{~h}$ ) after training. Ratio of pMAPK to tMAPK is plotted in the bar graph. pMAPK ratio elevates within the first $10 \mathrm{~min}$, then returns to basal level after $30 \mathrm{~min}\left(1 \mathrm{~min}: t_{(16)}=2.859, p=0.0114 ; 3 \mathrm{~min}: t_{(12.97)}=2.638, p=\right.$ $0.0205 ; 10 \mathrm{~min}: t_{(12.81)}=2.628, p=0.0211 ; 30 \mathrm{~min}: t_{(14.71)}=0.3083, p=0.7622 ; 1 \mathrm{~h}: t_{(15.59)}=0.2725, p=0.7888 ; 3 \mathrm{~h}: t_{(15.98)}$ $=0.4946, p=0.6276, n=8-9) . B$, The experience-dependent MAPK activation disappears in Dop 1R2-attenuated flies. Western blot shows PMAPK and tMAPK in naive (N) and conditioned ( $3 \mathrm{~min}$ and $10 \mathrm{~min}$ after conditioning) transgenetic flies. Ratio of pMAPK to tMAPK is shown. PMAPK ratio relatively increases after conditioning in genetic control flies, but not in the Dop1R2attenuated flies (R57C10-GAL4/+: $F_{(2,21)}=6.438, p=0.0066 ;+/$ UAS-Dop1R2.RNAi: $F_{(2,21)}=9.013, p=0.0015 ; R 57 C 10$ GAL4/UAS-Dop1R2.RNAi: $\left.F_{(2,21)}=0.007, p>0.9, n=8\right)$. Bars and error bars, mean $\pm S E M$, respectively. Each bar representing conditioned flies are compared with the corresponding naive controls. ${ }^{*} p<0.05,{ }^{* *} p<0.01$. ns, Not significant $(p>0.05)$.

A
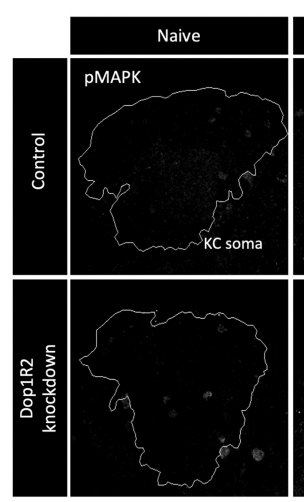

B

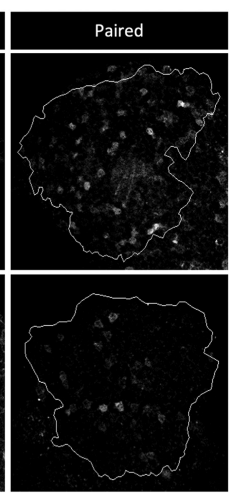

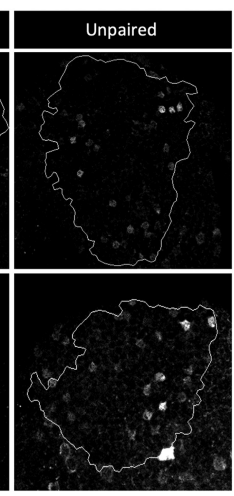

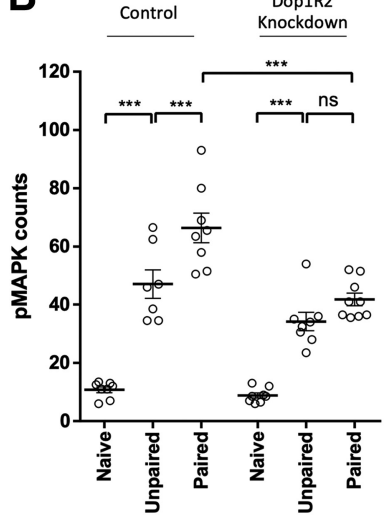

Figure 5. Paired presentation of sugar reward and an odor induces Dop1R2-dependent MAPK phosphorylation in KCs. $\boldsymbol{A}$, pMAPK immunohistochemistry at the KC soma region (outlined) is shown in naive (left), 3 min after the unpaired presentation of sugar reward and an odor (middle) or 3 min after the paired presentation (right). KCs are labeled by mCD8::GFP and Dop1R2 is knocked down in KCS (Control in top, UAS-mCD8::GFP/+;MB010B-GAL4/+;Dop1R2 knockdown in bottom, UAS-mCD8::GFP/+ ; MB010B-GAL4/UAS-Dop1R2.RNAi). B , The number of pMAPK-positive KCs (pMAPK counts) increases in a coincidental and Dop1R2dependent manner. Unpaired presentation of sugar reward and an odor increases pMAPK counts compared with the naive in both control $\left(F_{(5,42)}=47.2, p<0.0001\right.$; naive vs unpaired: $\left.p<0.0001\right)$, and the Dop 1R2 knockdown flies (naive vs unpaired: $p<$ 0.0001). A further increase of pMAPK in the paired group is observed in control flies (paired vs unpaired: $p=0.0009$ ), but not in Dop 1R2 knockdown flies (paired vs unpaired: $p=0.3865$ ). A significant difference in pMAPK counts between the control and the Dop1R2 knockdown flies is detected after paired presentation (control vs Dop1R2 knockdown: $p<0.0001$ ). $n=7-9$. pMAPK counts per hemispheres is shown. The sample images with median pMAPK counts in each group are selected for $\boldsymbol{A}$. Bars and error bars, mean \pm SEM, respectively. ${ }^{* *} p<0.001$. ns, Not significant $(p>0.05)$.

KCs, we found that the paired presentation of sugar reward and an odor induces MAPK phosphorylation in KCs, compared with the unpaired group (Fig. 5A,B). Strikingly, associative training failed to induce MAPK phosphorylation in KCs where Dop1R2 expression is downregulated (Fig. $5 A, B$ ). These results revealed that the association of sugar reward and an odor activates MAPK in KCs, in a Dop1R2-dependent manner.

Expression of constitutively active Raf rescues the LTM deficit caused by the Dop1R2 knockdown

If the Raf/MAPK pathway is the intracellular target of Dop1R2 signaling for appetitive LTM, activation of Raf could surrogate Dop1R2 signaling. To examine this hypothesis, we transiently expressed a truncated form of Raf lacking the regulatory domain (Raf.GOF; Brand and Perrimon, 1994) in KCs together with attenuating Dop1R2-expression. Intriguingly, the $24 \mathrm{~h}$ memory deficit in Dop $1 R 2$ knockdown flies was rescued by overexpressing Raf.GOF (Fig. 6A; MBSW-GAL4, UAS-Raf.GOF/UAS-Dop1R2.RNA). In contrast, the impairment due to the Dop1R1 knockdown was not rescued by Raf.GOF expression (Fig. 6A; MBSWGAL4, UAS-Raf.GOF/UAS-Dop1R1.RNA). Together, these observations reveal that Raf/ MAPK is a pivotal downstream effector of Dop1R2, but not Dop1R1, during appetitive memory stabilization.

In the course of the experiments, we noticed that overexpression of Raf.GOF alone reduced LTM but not STM (Fig. $6 A, B)$, a similar memory impairment to the knockdown of Raf (Fig. 2). In the previous experiments, we used the standard differential conditioning protocol, in which one of the two odors presented during conditioning was not paired with the sugar reward, and therefore serves as the reference odor (Tempel et al., 1983). As the presentation of an unpaired odor during training has been shown to be critical for odor discrimination (Barth et al., 2014; Schleyer et al., 2018), we simplified the paradigm by presenting only one odor in training and test, referred to as singleodor learning here (Fig. 6C). Interestingly, overexpression of Raf.GOF in KCs did not lead to $24 \mathrm{~h}$ memory deficit in the protocol without a reference odor, while knockdown of Raf or Dop1R2 did as in differential learning (Fig. 6D-F). Strikingly, expression of Raf.GOF in KCs significantly rescued the memory impairment of transient Dop1R2 knockdown in single-odor conditioning as well, suggesting that activation of Dop1R2 and Raf is critical for associative reward memory (Fig. 6G). A previous report showed that the expression of Raf.GOF does not significantly alter the basal pMAPK level (Zhang et al., 2018), but we observed a behavioral defect in the differential conditioning (Fig. 6A). These results imply that the titration of the Raf activity is critical for processes relevant to an unpaired reference odor. 
A

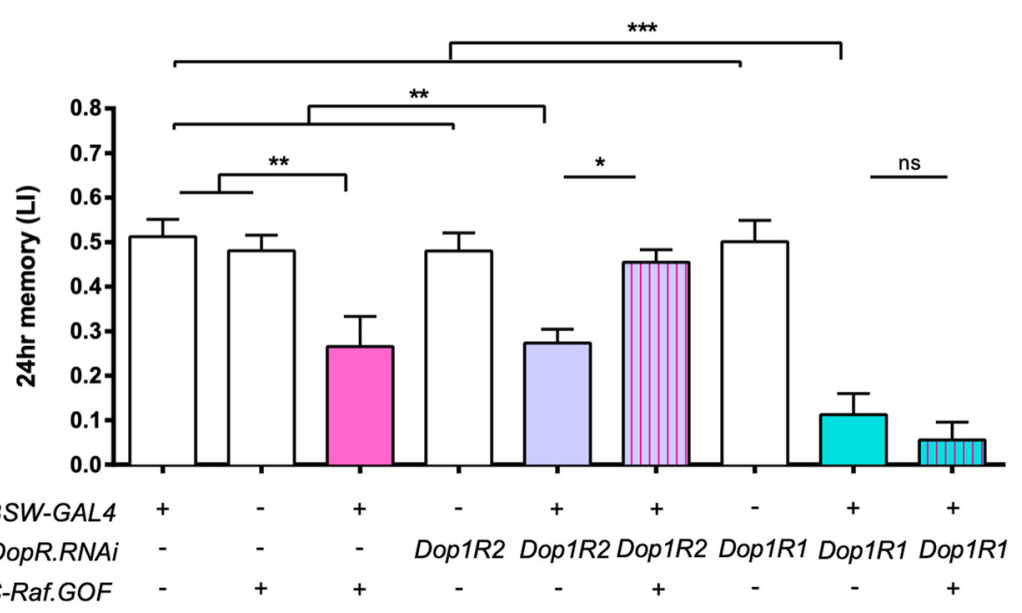

C

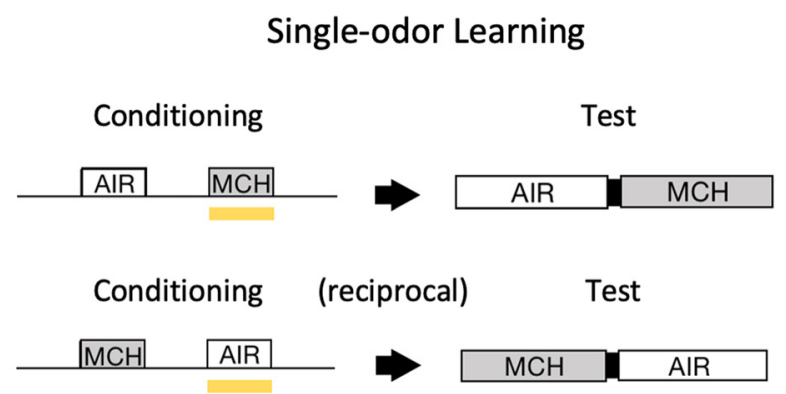

\section{Sucrose reward}

E

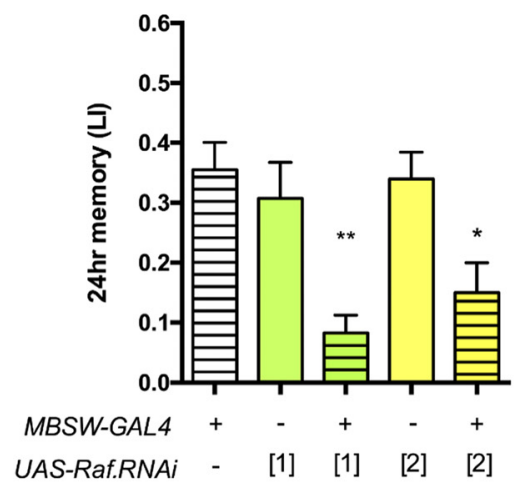

F

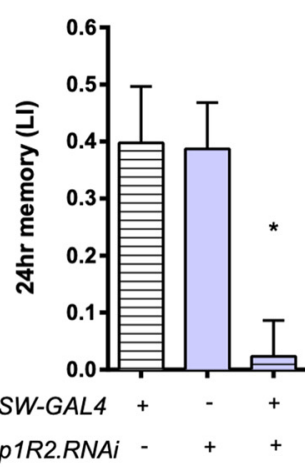

B

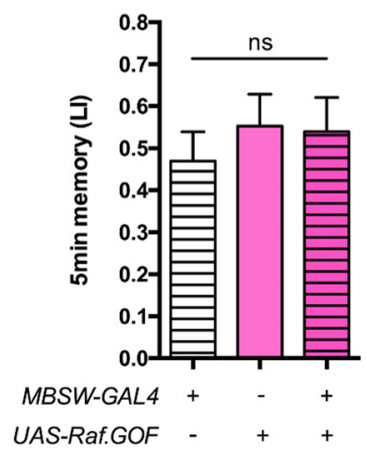

D

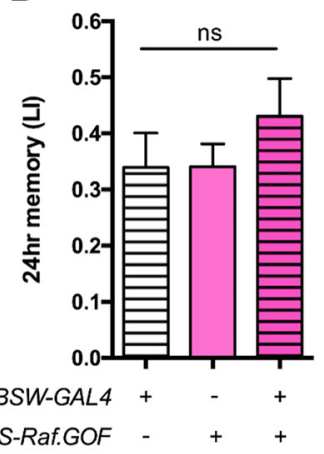

Figure 6. Expression of constitutively active Raf rescues the LTM deficit caused by the Dop 1R2 knockdown. A, Attenuation of Dop 1R2 or Dop 1R1 expression, as well as the overexpression of active $\operatorname{Raf}\left(\right.$ Raf.GOF) in adult KCs impairs $24 \mathrm{~h}$ memory $\left(F_{(8,125)}=14.68, p<0.0001\right.$; MBSW-GAL4/UAS-Dop1R2.RNAi vs GAL4 and UAS controls: $p=0.0011$ and $p=0.0083$, respectively; MBSW-GAL4/ UAS-Dop1R1.RNAi vs controls, $p<0.0001$ to both;MBSW-GAL4/UAS-Raf.GOFvs controls: $p=0.0004$ and $p=0.0034$ ). The deficit in $24 \mathrm{~h}$ memory caused by Dop1R2 attenuation is rescued by the overexpression of Raf.GOF, whereas that by Dop1R1 attenuation is not (MBSW-GAL4, UAS-Raf.GOF/UAS-Dop1R2.RNAi vs MBSW-GAL4/UAS-Dop1R2.RNAi: $p=0.0275 ;$ MBSW-GAL4, Raf.GOF/ Dop1R1.RNAivs MBSW-GAL4/Dop1R1.RNAi: $p>0.9) . n=12-17 . B$, Overexpression of Raf.GOFdoes not alter 5 min memory $\left(F_{(2,27)}=0.3734, p=0.6919, n=9-11\right) . C$, Schematics of single-odor learning by using only $M C H$. (Note that all preceding figures show the results of differential learning using two odors, $M C H$ and octan-3-ol.) $\boldsymbol{D}-\boldsymbol{F}$, In single-odor learning, the overexpression of Raf.GOF does not impair $24 \mathrm{~h}$ memory ( $\left.\boldsymbol{D}: F_{(2,32)}=0.8472, p=0.4380, n=13-14\right)$, while knockdown of Raf or Dop1R2 does $\left(E: F_{(4,36)}=6.789, p=0.0004 ;\right.$ MBSW-GAL4/UAS-Raf.RNAi[1] vs controls: $p=0.0008$ and $p=0.008$, respectively; MBSW-GAL4/UAS-Raf.RNAi[2] vs controls: $p=0.0140$ and $p=0.0311$, respectively; F: $p=0.0062, n=8-12$ ). G, In single-odor learning, the overexpression of Raf.GOF rescues the impaired $24 \mathrm{~h}$ memory caused by Dop 1R2-attenuation in $\mathrm{KCs}\left(F_{(5,8)}=7.077, p<0.0001\right.$; MBSW-GAL4, UAS-Raf.GOF/UAS-Dop1R2.RNAivs MBSW-GAL4/UASDop1R2.RNAi: $p=0.0222 ;$ MBSW-GAL4/UAS-Raf.GOFvs controls: $p>0.9$ and $p=0.4995$, respectively; MBSW-GAL4/UAS-Dop1R2.RNAivs controls: $p=0.0004$ and $p=0.0011$, respectively, $n=$ $8-11)$. Bars and error bars, mean \pm SEM, respectively. ${ }^{*} p<0.05,{ }^{* *} p<0.01,{ }^{* * *} p<0.001$. ns, Not significant $(p>0.05)$.

\section{Discussion}

Our study supports the idea that Dop1R2 signaling through the Raf/MAPK pathway in KCs is critical in stabilizing appetitive memory. This could be achieved through acquisition or consolidation of appetitive LTM. How is post-training Dop1R2 signal- ing triggered in this context? Accumulating evidence implies that Dop1R2 detects the basal dopamine release after learning (Berry et al., 2012; Musso et al., 2015; Ichinose et al., 2017). In aversive olfactory learning, the post-training enhancement of the oscillatory activity of MB-projecting DANs (MB-MP1 and MB-MV1) 
underlies LTM consolidation (Plaçais et al., 2012), and Dop1R2 in KCs is responsible for detecting the enhanced dopamine signals (Plaçais et al., 2017). This signaling is also reported to mediate forgetting early labile memory (Berry et al., 2012), suggesting distinct neural mechanisms to regulate memories with different temporal dynamics. In appetitive learning, Dop1R2 is suggested to be the mediator of the oscillating DANs, which represent the energy value of the reward and consolidate LTM (Musso et al., 2015; Pavlowsky et al., 2018). Collectively, after conditioning Dop1R2 signaling upon specific reinforcement input is a conserved mechanism to stabilize LTM. As MB-projecting DANs are also engaged in conveying reward information during memory acquisition (Burke et al., 2012; Liu et al., 2012; Yamagata et al., 2015), the Dop1R2/Raf/MAPK pathway might additionally be involved during the acquisition of LTM.

In contrast to the well characterized receptor tyrosine kinase signaling, it is rather unexpected to find the Raf/MAPK pathway as a downstream target of Dop1R2, a G-protein-coupled receptor (Figs. 5, 6). Dop1R2 was recently shown to have a preferential affinity to the $\mathrm{G} \alpha$ q subunit to elicit a robust intracellular $\mathrm{Ca}^{2+}$ increase upon ligand stimulation in KCs (Himmelreich et al., 2017; Handler et al., 2019). There are multiple lines of biochemical evidence suggesting that $\mathrm{G} \alpha_{\mathrm{q}}$-dependent $\mathrm{Ca}^{2+}$ signals could trigger several pathways, such as small GTPase Rap1, protein kinase C, or Ras, to activate Raf (Liebmann, 2001; Goldsmith and Dhanasekaran, 2007). Furthermore, some reports suggested that calcium influx through $\mathrm{N}$-methyl-D-aspartate receptor induces transient MAPK phosphorylation (English and Sweatt, 1996; Atkins et al., 1998). Hence, intracellular $\mathrm{Ca}^{2+}$ might be the key second-messenger system to link Dop1R2 and Raf/MAPK in appetitive LTM.

We found that MAPK has a pivotal role to stabilize appetitive memory in KCs (Fig. 3). MAPK signaling is known to regulate different cellular processes ranging from cytoskeletal dynamics to transcriptional modulation (Pullikuth and Catling, 2007; Giese and Mizuno, 2013). In Drosophila, a recent work unveiled that MAPK stabilizes presynaptic structural changes in KCs upon associative training with electric shocks, reportedly by changing the activity of an actin cytoskeleton regulator (Zhang et al., 2018). Such MAPK-induced cytoskeletal change might also occur in appetitive learning. Alternatively, a recent study showed that LTM consolidation involves MAPK translocation to the nuclei in $\mathrm{KCs}$ (Li et al., 2016). Consistently, it is reported that MAPK activates transcription factors like c-Fos and cAMP response element-binding protein (CREB) in KCs to form aversive LTM (Miyashita et al., 2018). Appetitive LTM is also dependent on CREB in KCs (Krashes and Waddell, 2008; Widmer et al., 2018). Collectively, we propose that MAPK stabilizes appetitive memory by regulating these transcription factors. Future investigation on the downstream of the MAPK pathway should reveal the newly transcribed genes for memory stabilization.

\section{References}

Aso Y, Hattori D, Yu Y, Johnston RM, Iyer NA, Ngo TT, Dionne H, Abbott LF, Axel R, Tanimoto H, Rubin GM (2014) The neuronal architecture of the mushroom body provides a logic for associative learning. Elife 3:e04577.

Atkins CM, Selcher JC, Petraitis JJ, Trzaskos JM, Sweatt JD (1998) The MAPK cascade is required for mammalian associative learning. Nat Neurosci 1:602-609.

Barth J, Dipt S, Pech U, Hermann M, Riemensperger T, Fiala A (2014) Differential associative training enhances olfactory acuity in Drosophila melanogaster. J Neurosci 34:1819-1837.
Berry JA, Cervantes-Sandoval I, Nicholas EP, Davis RL (2012) Dopamine is required for learning and forgetting in Drosophila. Neuron 74:530-542.

Brand AH, Perrimon N (1994) Raf acts downstream of the EGF receptor to determine dorsoventral polarity during Drosophila oogenesis. Genes Dev 8:629-639.

Burke CJ, Huetteroth W, Owald D, Perisse E, Krashes MJ, Das G, Gohl D, Silies M, Certel S, Waddell S (2012) Layered reward signalling through octopamine and dopamine in Drosophila. Nature 492:433-437.

Busto GU, Cervantes-Sandoval I, Davis RL (2010) Olfactory learning in Drosophila. Physiology (Bethesda) 25:338-346.

Colomb J, Kaiser L, Chabaud MA, Preat T (2009) Parametric and genetic analysis of Drosophila appetitive long-term memory and sugar motivation. Genes Brain Behav 8:407-415.

Croset V, Treiber CD, Waddell S (2018) Cellular diversity in the Drosophila midbrain revealed by single-cell transcriptomics. Elife 7:e34550.

English JD, Sweatt JD (1996) Activation of p42 mitogen-activated protein kinase in hippocampal long term potentiation. J Biol Chem 271: 24329-24332.

Giese KP, Mizuno K (2013) The roles of protein kinases in learning and memory. Learn Mem 20:540-552.

Goldsmith ZG, Dhanasekaran DN (2007) G protein regulation of MAPK networks. Oncogene 26:3122-3142.

Handler A, Graham TGW, Cohn R, Morantte I, Siliciano AF, Zeng J, Li Y, Ruta V (2019) Distinct dopamine receptor pathways underlie the temporal sensitivity of associative learning. Cell 178:60-75.e19.

Hearn MG, Ren Y, McBride EW, Reveillaud I, Beinborn M, Kopin AS (2002) A drosophila dopamine 2-like receptor: molecular characterization and identification of multiple alternatively spliced variants. Proc Natl Acad Sci U S A 99:14554-14559.

Himmelreich S, Masuho I, Berry JA, MacMullen C, Skamangas NK, Martemyanov KA, Davis RL (2017) Dopamine receptor DAMB signals via gq to mediate forgetting in Drosophila. Cell Rep 21:2074-2081.

Ichinose T, Tanimoto H (2016) Dynamics of memory-guided choice behavior in Drosophila. Proc Jpn Acad Ser B Phys Biol Sci 92:346-357.

Ichinose T, Aso Y, Yamagata N, Abe A, Rubin GM, Tanimoto H (2015) Reward signal in a recurrent circuit drives appetitive long-term memory formation. Elife 4:e10719.

Ichinose T, Tanimoto H, Yamagata N (2017) Behavioral modulation by spontaneous activity of dopamine neurons. Front Syst Neurosci 11:88.

Jenett A, Rubin GM, Ngo TT, Shepherd D, Murphy C, Dionne H, Pfeiffer BD, Cavallaro A, Hall D, Jeter J, Iyer N, Fetter D, Hausenfluck JH, Peng H, Trautman ET, Svirskas RR, Myers EW, Iwinski ZR, Aso Y, DePasquale GM, et al. (2012) A GAL4-driver line resource for Drosophila neurobiology. Cell Rep 2:991-1001.

Kondo S, Takahashi T, Yamagata N, Imanishi Y, Katow H, Hiramatsu S, Lynn K, Abe A, Kumaraswamy A, Tanimoto H (2020) Neurochemical organization of the Drosophila brain visualized by endogenously tagged neurotransmitter receptors. Cell Rep 30:284-297.e5.

Krashes MJ, Waddell S (2008) Rapid consolidation to a radish and protein synthesis-dependent long-term memory after single-session appetitive olfactory conditioning in Drosophila. J Neurosci 28:3103-3113.

Krüttner S, Traunmüller L, Dag U, Jandrasits K, Stepien B, Iyer N, Fradkin LG, Noordermeer JN, Mensh BD, Keleman K (2015) Synaptic Orb2A bridges memory acquisition and late memory consolidation in Drosophila. Cell Rep 11:1953-1965.

Leicht DT, Balan V, Kaplun A, Singh-Gupta V, Kaplun L, Dobson M, Tzivion G (2007) Raf kinases: function, regulation and role in human cancer. Biochim Biophys Acta 1773:1196-1212.

Li Q, Zhang X, Hu W, Liang X, Zhang F, Wang L, Liu ZJ, Zhong Y (2016) Importin-7 mediates memory consolidation through regulation of nuclear translocation of training-activated MAPK in Drosophila. Proc Natl Acad Sci U S A 113:3072-3077.

Liebmann C (2001) Regulation of MAP kinase activity by peptide receptor signalling pathway: paradigms of multiplicity. Cell Signal 13:777-785.

Liu C, Plaçais PY, Yamagata N, Pfeiffer BD, Aso Y, Friedrich AB, Siwanowicz I, Rubin GM, Preat T, Tanimoto H (2012) A subset of dopamine neurons signals reward for odour memory in Drosophila. Nature 488:512516.

Mao Z, Roman G, Zong L, Davis RL (2004) Pharmacogenetic rescue in time and space of the rutabaga memory impairment by using gene-switch. Proc Natl Acad Sci U S A 101:198-203.

Miyashita T, Kikuchi E, Horiuchi J, Saitoe M (2018) Long-term memory 
engram cells are established by c-Fos/CREB transcriptional cycling. Cell Rep 25:2716-2728.e3.

Moressis A, Friedrich AR, Pavlopoulos E, Davis RL, Skoulakis EM (2009) A dual role for the adaptor protein DRK in Drosophila olfactory learning and memory. J Neurosci 29:2611-2625.

Musso PY, Tchenio P, Preat T (2015) Delayed dopamine signaling of energy level builds appetitive long-term memory in Drosophila. Cell Rep 10:1023-1031.

Mustard JA, Beggs KT, Mercer AR (2005) Molecular biology of the invertebrate dopamine receptors. Arch Insect Biochem Physiol 59:103-117.

Nagai T, Nakamuta S, Kuroda K, Nakauchi S, Nishioka T, Takano T, Zhang X, Tsuboi D, Funahashi Y, Nakano T, Yoshimoto J, Kobayashi K, Uchigashima M, Watanabe M, Miura M, Nishi A, Kobayashi K, Yamada K, Amano M, Kaibuchi K (2016a) Phosphoproteomics of the dopamine pathway enables discovery of Rap1 activation as a reward signal in vivo. Neuron 89:550-565.

Nagai T, Yoshimoto J, Kannon T, Kuroda K, Kaibuchi K (2016b) Phosphorylation signals in striatal medium spiny neurons. Trends Pharmacol Sci $37: 858-871$.

O'Carroll CM, Martin SJ, Sandin J, Frenguelli B, Morris RG (2006) Dopaminergic modulation of the persistence of one-trial hippocampusdependent memory. Learn Mem 13:760-769.

Pagani MR, Oishi K, Gelb BD, Zhong Y (2009) The phosphatase SHP2 regulates the spacing effect for long-term memory induction. Cell 139: 186-198.

Pavlowsky A, Schor J, Plaçais PY, Preat T (2018) A GABAergic feedback shapes dopaminergic input on the Drosophila mushroom body to promote appetitive long-term memory. Curr Biol 28:1783-1793.e4.

Pfeiffer BD, Ngo TT, Hibbard KL, Murphy C, Jenett A, Truman JW, Rubin GM (2010) Refinement of tools for targeted gene expression in Drosophila. Genetics 186:735-755.

Plaçais PY, Trannoy S, Isabel G, Aso Y, Siwanowicz I, Belliart-Guérin G, Vernier P, Birman S, Tanimoto H, Preat T (2012) Slow oscillations in two pairs of dopaminergic neurons gate long-term memory formation in Drosophila. Nat Neurosci 15:592-599.

Plaçais PY, de Tredern É, Scheunemann L, Trannoy S, Goguel V, Han KA, Isabel G, Preat T (2017) Upregulated energy metabolism in the Drosophila mushroom body is the trigger for long-term memory. Nat Commun 8:15510.

Puig MV, Antzoulatos EG, Miller EK (2014) Prefrontal dopamine in associative learning and memory. Neuroscience 282:217-229.

Pullikuth AK, Catling AD (2007) Scaffold mediated regulation of MAPK signaling and cytoskeletal dynamics: a perspective. Cell Signal 19: 1621-1632.
Qin H, Cressy M, Li W, Coravos JS, Izzi SA, Dubnau J (2012) Gamma neurons mediate dopaminergic input during aversive olfactory memory formation in Drosophila. Curr Biol 22:608-614.

Roman G, Endo K, Zong L, Davis RL (2001) P[Switch], a system for spatial and temporal control of gene expression in Drosophila melanogaster. Proc Natl Acad Sci U S A 98:12602-12607.

Rossato JI, Bevilaqua LR, Izquierdo I, Medina JH, Cammarota M (2009) Dopamine controls persistence of long-term memory storage. Science 325:1017-1020.

Schleyer M, Fendt M, Schuller S, Gerber B (2018) Associative learning of stimuli paired and unpaired with reinforcement: evaluating evidence from maggots, flies, bees, and rats. Front Psychol 9:1494.

Scholz-Kornehl S, Schwärzel M (2016) Circuit analysis of a Drosophila dopamine type 2 receptor that supports anesthesia-resistant memory. J Neurosci 36:7936-7945.

Srivastava DP, Yu EJ, Kennedy K, Chatwin H, Reale V, Hamon M, Smith T, Evans PD (2005) Rapid, nongenomic responses to ecdysteroids and catecholamines mediated by a novel Drosophila G-protein-coupled receptor. J Neurosci 25:6145-6155.

Sugamori KS, Demchyshyn LL, McConkey F, Forte MA, Niznik HB (1995) A primordial dopamine D1-like adenylyl cyclase-linked receptor from Drosophila melanogaster displaying poor affinity for benzazepines. FEBS Lett 362:131-138.

Takeuchi T, Duszkiewicz AJ, Sonneborn A, Spooner PA, Yamasaki M, Watanabe M, Smith CC, Fernández G, Deisseroth K, Greene RW, Morris RG (2016) Locus coeruleus and dopaminergic consolidation of everyday memory. Nature 537:357-362.

Tempel BL, Bonini N, Dawson DR, Quinn WG (1983) Reward learning in normal and mutant Drosophila. Proc Natl Acad Sci U S A 80:1482-1486.

Vogt K, Schnaitmann C, Dylla KV, Knapek S, Aso Y, Rubin GM, Tanimoto H (2014) Shared mushroom body circuits underlie visual and olfactory memories in Drosophila. Elife 3:e02395.

Widmer YF, Fritsch C, Jungo MM, Almeida S, Egger B, Sprecher SG (2018) Multiple neurons encode CrebB dependent appetitive long-term memory in the mushroom body circuit. Elife 7:e39196.

Yamagata N, Ichinose T, Aso Y, Plaçais PY, Friedrich AB, Sima RJ, Preat T, Rubin GM, Tanimoto H (2015) Distinct dopamine neurons mediate reward signals for short- and long-term memories. Proc Natl Acad Sci U S A 112:578-583.

Zhang X, Li Q, Wang L, Liu ZJ, Zhong Y (2018) Active protection: learningactivated Raf/MAPK activity protects labile memory from Raclindependent forgetting. Neuron 98:142-155.e4. 